\title{
COVID-19 disease diagnosis from paper-based ECG trace image data using a novel convolutional neural network model
}

\author{
Emrah Irmak ${ }^{1}[$
}

Received: 24 August 2021 / Accepted: 6 January 2022 / Published online: 12 January 2022

(c) Australasian College of Physical Scientists and Engineers in Medicine 2022

\begin{abstract}
Clinical reports show that COVID-19 disease has impacts on the cardiovascular system in addition to the respiratory system. Available COVID-19 diagnostic methods have been shown to have limitations. In addition to current diagnostic methods such as low-sensitivity standard RT-PCR tests and expensive medical imaging devices, the development of alternative methods for the diagnosis of COVID-19 disease would be beneficial for control of the COVID-19 pandemic. Further, it is important to quickly and accurately detect abnormalities caused by COVID-19 on the cardiovascular system via ECG. In this study, the diagnosis of COVID-19 disease is proposed using a novel deep Convolutional Neural Network model by using only ECG trace images created from ECG signals of COVID-19 infected patients based on the abnormalities caused by the COVID-19 virus on the cardiovascular system. An overall classification accuracy of $98.57 \%, 93.20 \%, 96.74 \%$ and AUC value of 0.9966 , $0.9771,0.9905$ is achieved for COVID-19 vs. Normal, COVID-19 vs. Abnormal Heartbeats, COVID-19 vs. Myocardial Infarction binary classification tasks, respectively. In addition, an overall classification accuracy of $86.55 \%$ and $83.05 \%$ is achieved for COVID-19 vs. Abnormal Heartbeats vs. Myocardial Infarction and Normal vs. COVID-19 vs. Abnormal Heartbeats vs. Myocardial Infarction multi-classification tasks. This study is believed to have great potential to speed up the diagnosis and treatment of COVID-19 patients, saving clinicians time and facilitating the control of the pandemic.
\end{abstract}

Keywords Cardiovascular diseases diagnosis · Convolutional neural networks · COVID-19 diagnosis · Deep learning · Electrocardiography $\cdot$ Machine learning

\section{Introduction}

Coronaviruses are large family of viruses that can cause various diseases in animals or humans. The Novel Coronavirus Disease (COVID-19) first appeared in Wuhan Province, China towards the end of 2019 and has spread around the world at an unprecedented speed [1]. The most common symptoms are fever, cough and shortness of breath. In severe cases, pneumonia, severe respiratory failure, renal failure and death may develop. As of 16 June 2021, the total number of cases in the world has exceeded 176 million and the total number of deaths has exceeded 4 million people [2]. Although it is known that there is no effective medicine for the COVID-19 disease, which the World Health

Emrah Irmak

emrah.irmak@alanya.edu.tr

1 Electrical-Electronics Engineering Department, Alanya Alaaddin Keykubat University, 07425 Alanya, Antalya, Turkey
Organization (WHO) declared as a pandemic on March 11, 2020 [3], there have been promising developments in vaccine studies. Scientific reports have announced that the effectiveness of the vaccines is quite high and $20.8 \%$ of the world population has received at least one dose of a COVID-19 vaccine as of writing this paper [4].

Considering the high contagious nature of the virus, a fast and accurate diagnostic method is undoubtedly critical for controlling the pandemic. Throughout the pandemic, the diagnosis of COVID-19 disease has been done with tests known as Reverse Transcription Polymerase Chain reactions (RT-PCR) based on the analysis of swabs taken from the throat and nose. The lack of sensitivity of these tests has led the scientific world to alternative diagnostic methods [5]. As a result of intensive studies and radiological imaging science, it has been observed that the virus has effects such as ground-glass opacity, crazy paving pattern, consolidation, vascular enlargement, lower-lobe involvement and bilateral infiltration in the lungs [6]. Therefore, radiological imaging techniques, especially MRI and CT, have been 
used as alternative tools for COVID-19 disease diagnosis. The overall accuracy and sensitivity of radiological imaging techniques such as X-ray and CT for COVID-19 disease diagnosis is quite high as being over 95\% [7-9]. However, access to radiological imaging devices is not easy and cheap, especially in remote, rural and underdeveloped regions. Moreover, diagnosis methods based on radiological imaging techniques seem to be slow in comparison to the very rapid spread of the novel coronavirus. The COVID-19 is actually a pneumonia virus and the changes it creates in the lungs are very similar to the changes caused by communityacquired pneumonia viruses, which is another disadvantage that makes radiological imaging-based diagnostic methods difficult to accurately diagnose COVID-19 disease. Existing diagnostic methods have these shortcomings, which show the necessity of fast and also effective alternative diagnostic methods. The medical science world reveals that this dangerous virus affects many organs and systems in the human body and causes changes in the related system $[10,11]$. An important system in which the virus affects its normal functioning is the cardiovascular system [12-15]. ECG physiological signals, on the other hand, are a very good indicator to show abnormal conditions occurring in the cardiovascular system. This paper investigates the possibility of COVID-19 disease diagnosis from paper-based ECG data using Deep Learning (DL) phenomenon taking into account the promising ECG features such as accessibility, reliability, low cost, real-time monitoring, harmlessness etc. The proposed method in this paper is believed to be an alternative highaccurate and effective method to fill the gap for COVID-19 disease diagnosis.

DL based image processing methods have been proven to be very effective and useful, especially for medical diagnosis and treatment. Therefore, DL based image processing techniques have been used effectively in various forms since the beginning of the pandemic, both for COVID-19 disease diagnosis and treatment purposes. For example, Convolutional Neural Networks model (CNN), which is considered one of the most successful DL methods, has been successfully performed for COVID-19 disease detection using chest X-ray images. To begin with, Togacar et al. have succeeded to detect COVID-19 disease with an accuracy of $99.27 \%$ using chest X-ray images and CNN method [16]. In another DL based method using chest X-ray images, Apostolopoulos et al. have achieved COVID-19 disease detection with an accuracy of $96.78 \%$ [17]. Irmak E. has used quite large publicly available dataset and a novel CNN model for COVID-19 disease detection as well as COVID-19 vs. Community Acquired Pneumonia vs. Healthy multi-classification task. He has obtained $98.92 \%$ accuracy for binary classification and $98.27 \%$ accuracy for multi-classification, respectively. The COVID-19 disease detection from chest
X-ray images has been effectively studied in another DL based image processing methods. For example, in [18], Generative Adversial Network (GAN) and Deep Transfer Learning have been used and a high accuracy rate such as $99.9 \%$ has been obtained. COVID-19 disease diagnosis has been successfully performed using DL methods from CT images as well. For instance, Li et al. have developed a fully automatic method to detect COVID-19 using chest CT images [5]. Singh et al. was another researcher group who studied COVID-19 disease diagnosis from chest CT images using CNN method [19]. They have used multiobjective differential evolution (MODE) technique for parameter tuning and obtained a good accuracy rate. Chen et al. have constructed a system based on DL for detecting COVID-19 disease on high resolution CT images [20]. They have used quite high number of data and diagnosed COVID-19 disease with $95.24 \%$ accuracy. Radiological imaging and DL based image processing techniques have also been utilized for COVID-19 disease quantification and risk stratification within last past year of pandemic. For example, Chaganti et al. have suggested to use a DL and deep reinforcement-based method to compute the measures of COVID-19 disease severity [21]. In this study, the Percentage of Opacity (PO) and Lung Severity Score (LSS) have been computed from abnormalities such as ground glass opacity and consolidations associated with COVID-19 disease. The combined severity measures have been fed into DL model and COVID-19 patients have been classified as severe or non-severe cases. Chassagnon et al. have staged COVID-19 infected patients and predicted outcomes of COVID-19 disease using automatic DL based image processing method and lung CT images [22]. Irmak E. has also performed COVID-19 disease severity assessment from radiological images using novel CNN models [9]. The COVID19 infected patients have been successfully divided into Mild vs. Moderate vs. Severe vs. Critical severity groups with an overall accuracy of $95.52 \%$ and AUC value of 0.9873. In a successful retrospective study, $\mathrm{Li}$ et al. have proposed a DL based image processing model to quantitatively assess the disease severity and disease progression using chest CT images of COVID-19 patients [23]. Haung et al. have used a commercially available DL based tool to quantitatively predict the COVID-19 disease severity stages [24]. COVID-19 infected patients have been classified as Mild Type vs. Moderate Type vs. Severe Type vs. Critical Type using CT lung opacification percentages of the whole lung. Another important DL based COVID-19 disease severity assessment study has proposed staging COVID-19 infected patients on portable chest radiographs [25]. Prediction of lung disease severity scores has been performed using deep CNN model and have been compared with three expert chest radiologists ground truth 
scores. There are also important publications that investigated the usage of ECG data to detect COVID-19 disease. For example, Ozdemir et al. have proposed a DL based method to detect COVID-19 disease using ECG data [26]. They have obtained detection accuracy of $96.20 \%$. In another publication by Bergamaschi et al., the prognostic value of ECG in COVID-19 disease has been explored [12]. It has been concluded that there were powerful links between COVID-19 disease and ECG alterations. Rahman et al. have used pre-trained CNN methods to detect COVID-19 disease from ECG data [27]. They have found that the pre-trained DenseNet201 has outperformed other CNN models in COVID-19 disease diagnosis from ECG data. Another researcher group, Shi et al., have explored the characteristics and clinical significance of ECG in COVID-19 disease [28]. He et al. have reported that ECG changes can be hallmark of cardiac injury, which further showed the importance of usage of ECG data for COVID-19 disease diagnosis [29]. Churchill et al. have suggested that the ECG can be an essential diagnostic tool in COVID-19 disease detection by investigating the echocardiographic finding in patients with COVID-19 [30]. Finally, the study by Nemati et al. is an important systematic literature review for the researchers who are interested in the usage of ECG data to detect COVID-19 disease [13].

In this paper, a novel deep CNN model is proposed for COVID-19 disease diagnosis as well as COVID-19 vs. Abnormal Heartbeats binary classification, COVID-19 vs. Myocardial Infarction binary classification, COVID19 vs. Abnormal Heartbeats vs. Myocardial Infarction and Normal vs. COVID-19 vs. Abnormal Heartbeats vs. Myocardial Infarction multi-classification tasks from ECG trace images created from ECG signals acquired by non-invasive 12-lead ECG device from COVID-19 infected patients based on the abnormalities caused by the COVID-19 virus on the cardiovascular system. The remaining of this paper can be organized as follows. Section 2 explains the proposed Methodology which contains dataset and the proposed CNN model details. Section 3 is dedicated to Experimental Results whereas Sect. 4 is left for Discussions. Comparison of the proposed study with the state-of-art studies can also be found in this section. Finally, Sect. 5 concludes the paper.

\section{Methodology}

\section{Dataset and software-hardware environment}

The dataset used in this study contains the ECG trace images of patients suffering from COVID-19 and Cardiac diseases. To the best of author's knowledge, this dataset is quite a rare dataset such that it is the only publicly available dataset [31] until the writing of this paper. The dataset includes 1937 different patient's ECG data which was recorded using 12-lead with $500 \mathrm{~Hz}$ sample rate ECG Device "EDAN SERIES-3" installed in various clinical centers. After the manual review of the recorded ECG images by medical experts with experience in ECG, the dataset was divided into 5 distinct groups as shown in Table 1 . Now that the images were collected from different institutions and patients, they differ in standard and resolution. Therefore, not all the images are appropriate to be used in this study. Total number of images in each group in original dataset and in dataset that is used in this study is demonstrated in Table 1. This dataset is multiclass which means that a patient cannot have more than one class at the same time.

As can be seen in Table 1, the data is not balanced between the data groups. In addition to this, the number of images is not sufficient for an efficient DL based image processing study. Therefore, the dataset is augmented using scaling, translation and rotation to improve image classifier (CNN model) performance. The data augmentation is performed to reduce overfitting and memorizing problems as well as data imbalance problem. The number of images before and after data augmentation is demonstrated in Table 2. Figure 1 shows sample ECG trace images. All the experiments are conducted using Windows 10 (64-bit) operating system with Matlab R2019a software environment and NVIDIA GeForce GTX-850M GM107 GPU, Intel Core i7 5400 GPU 2.60 Ghz, 16.0 GB RAM hardware environment.

Table 1 Dataset description

\begin{tabular}{lcc}
\hline & $\begin{array}{l}\text { Number of images in the original } \\
\text { dataset }\end{array}$ & $\begin{array}{l}\text { Number of images } \\
\text { used in this study }\end{array}$ \\
\hline Normal & 859 & 713 \\
Myocardial infarction & 77 & 77 \\
Recovered myocardial infarction & 203 & 187 \\
Abnormal heartbeat & 548 & 500 \\
COVID-19 & 250 & 218 \\
\hline
\end{tabular}


Table 2 Number of images used in this study for each classification task before and after data augmentation

\begin{tabular}{llcl}
\hline & Classification task & $\begin{array}{l}\text { Number of images before data } \\
\text { augmentation }\end{array}$ & $\begin{array}{l}\text { Number of images } \\
\text { after data augmenta- } \\
\text { tion }\end{array}$ \\
\hline Task-1 & COVID-19 & 218 & 650 \\
& Normal & 713 & 720 \\
Task-2 & COVID-19 & 218 & 650 \\
& Abnormal heartbeats & 500 & 600 \\
Task-3 & COVID-19 & 218 & 230 \\
& Myocardial infarction & 77 & 230 \\
& COVID-19 & 218 & 650 \\
Task-5 & Abnormal heartbeats & 500 & 500 \\
& Myocardial infarction & 77 & 300 \\
& Normal & 713 & 720 \\
& COVID-19 & 218 & 650 \\
& Abnormal heartbeats & 500 & 500 \\
& Myocardial infarction & 77 & 300 \\
\hline
\end{tabular}

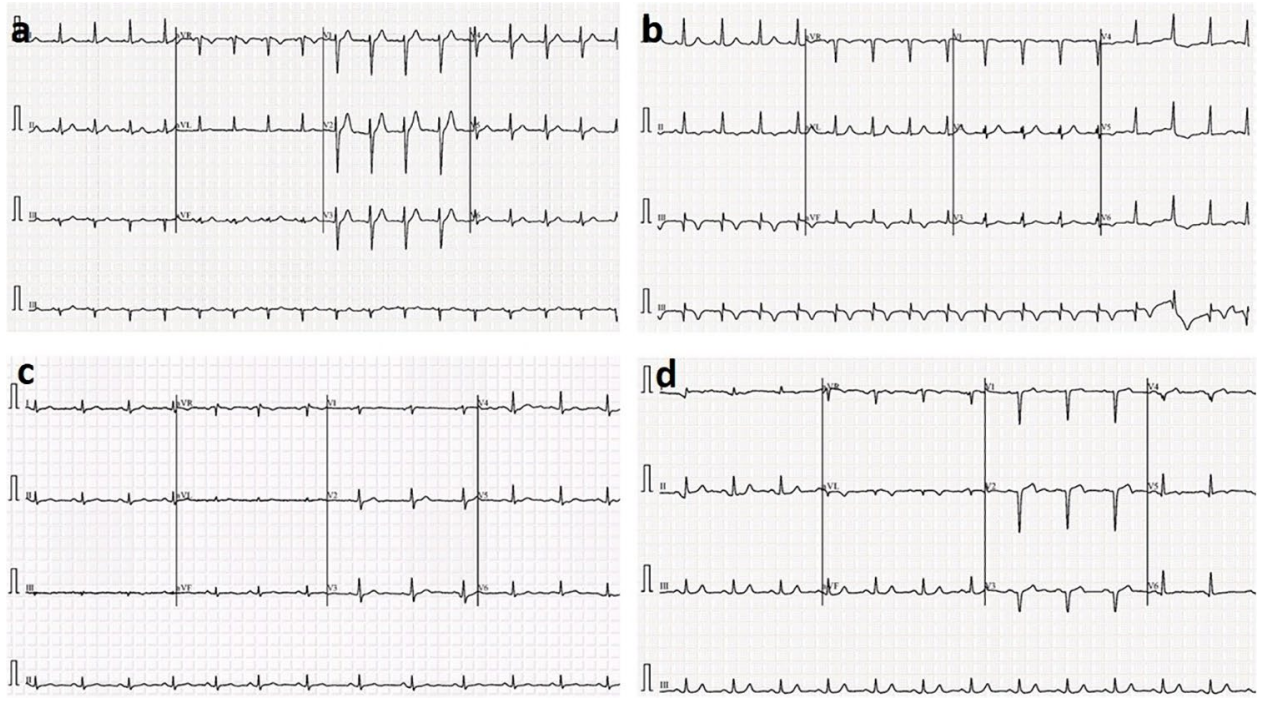

Fig. 1 Sample ECG trace images from dataset, a COVID19 ECG, b Normal ECG, c Abnormal Heartbeats ECG and d Myocardial ECG

\section{The proposed CNN model}

Current literature suggests that CNN models of DL are very efficient and successful for image classification problems [25, 32-34]. A typical CNN model consists of two parts: feature extraction and classification. CNN performs feature extraction and classification through sequentially trainable layers placed one after the other. Feature extraction part of the CNN generally includes the convolutional and pooling layers whereas the classification part includes the fully connected and classification layers. The training process is started by performing layer-by-layer operations just after CNN receives the input data. Finally, a final output is given to compare with the correct (actual) result. The differences between the estimated result and the actual result produce an error which is transferred to all weights by the back-propagation algorithm. Weights are updated with each iteration to reduce the error. In this study, the diagnosis of COVID-19 disease is proposed using a novel deep CNN model by using only ECG trace images created from ECG signals of COVID-19 infected patients based on the abnormalities caused by the COVID-19 virus on the cardiovascular system. The proposed CNN model has 20 weighted layers, in which there are one input layer, four convolutional layers, five rectified linear unit (ReLU) layers, one normalization layer, five max pooling layers, 2 fully-connected layers, one dropout layer and one classification layer as shown in Fig. 2. The input images are resized as $224 \times 224 \times 3$. The proposed CNN model in this study has been inspired from Visual Geometry Group (VGG-16) architecture [35]. In addition, the $\mathrm{CNN}$ architectures in the studies that were proposed for similar problems in the literature and that gave 
Fig. 2 The proposed $\mathrm{CNN}$ architecture successful results have been thoroughly examined while creating the CNN architecture in this paper. For example, in [39] a 17 layered CNN architecture has been successfully used for tumor grading. Moreover, tumor detection has been achieved with an optimum 12 layered $\mathrm{CNN}$ architecture in [40]. Irmak E. has proposed a 14 layered CNN architecture for COVID-19 disease detection [7]. Adaptive moment estimation algorithm (Adam) is used as optimizer in the proposed CNN model [36]. CNN hyper-parameters are tuned such that Mini-batch Size is $32[37,38]$ Learning Rate is $0.001[36,39]$, Momentum is 0.99 , and $l_{2}$ regularization is $0.0001[9,34]$.

\section{Performance evaluation metrics}

Performance evaluation metrics should be used to evaluate the quality of the classification process after any image classification problem. Otherwise, the performance evaluation of the solution would be qualitative rather than quantitative. Therefore, well-known performance evaluation metrics are used to assess the performance of the CNN model in this study. These performance evaluation metrics are Accuracy, Specificity, Sensitivity, and Precision. The Area Under the Curve of the Receiver Operating Characteristic (ROC) known as AUC of ROC curve is another important performance evaluation metric which is successfully used in image classification problems. This metric is also used in this study to assess the performance of the CNN models. Equation 1 shows the corresponding formulas associated with these performance evaluation metrics. TP, TN, FP and FN stand for True Positive, True Negative, False Positive and False Negative, respectively.

$$
\begin{aligned}
& \text { Accuracy }=\frac{\mathrm{TP}+\mathrm{TN}}{\mathrm{TP}+\mathrm{TN}+\mathrm{FP}+\mathrm{FN}} \\
& \text { Specificity }=\frac{\mathrm{TN}}{\mathrm{TN}+\mathrm{FP}} \\
& \text { Sensitivity }=\frac{\mathrm{TP}}{\mathrm{TP}+\mathrm{FN}} \\
& \text { Precision }=\frac{\mathrm{TP}}{\mathrm{TP}+\mathrm{FP}}
\end{aligned}
$$

\section{Experimental results}

Five different classification tasks are performed using a novel CNN model for COVID-19 diagnosis. Tenfold crossvalidation procedure is utilized for each experiment in this DL study in order to evaluate the performance of the study. The dataset is divided into 10 folds out of which 9 sets are used for training and the remaining one is used for testing. The experiments are repeated ten times. Classification performance for each task is evaluated for each fold, and the 
average classification performance of the model is calculated. High accuracies from training and validation phases are not meaningful without testing the trained and hyper parameter-tuned CNN on predicting unseen samples. Therefore, a test dataset is randomly assigned and separated along with training and validation datasets to test the performance of trained CNN on predicting samples; otherwise, the high accuracy may be due to biased dataset assignment. In the first classification task, COVID-19 vs. Normal classification task is performed. 650 COVID-19 infected patient ECG trace images and 720 healthy people ECG trace images are used for training and test of the CNN model. For this task, there are 1370 ECG trace images in total and these images are randomly separated as training, validation and test sets having the ratio of 60:20:20. In the second classification task, COVID-19 vs. Abnormal Heartbeats classification task is performed. 650 COVID-19 infected patient ECG trace images and 600 ECG trace images of abnormal heartbeats patients are used for training and test of the CNN model. For this task, there are 1250 ECG trace images in total and these images are randomly separated as training, validation and test sets having the ratio of 60:20:20. In the third classification task, COVID-19 vs. Myocardial Infarction classification task is performed. 230 COVID-19 infected patient ECG trace images and 230 ECG trace images of myocardial infarction patients are used for training and test of the CNN model. For this task, there are 460 ECG trace images in total and these images are randomly separated as training, validation and test sets having the ratio of 60:20:20. In the fourth classification task, COVID-19 vs. Abnormal Heartbeats vs. Myocardial Infarction multi-classification task is performed. 650 COVID-19 infected patient ECG trace images, 600 ECG trace images of abnormal heartbeats patients and $300 \mathrm{ECG}$ trace images of myocardial infarction patients are used for training and test of the CNN model. For this task, there are 1450 ECG trace images in total and these images are randomly separated as training, validation and test sets having the ratio of 60:20:20. In the fifth classification task, Normal vs. COVID-19 vs. Abnormal Heartbeats vs. Myocardial
Infarction multi-classification task is performed. 720 ECG trace images of healthy people, 650 COVID-19 infected patient ECG trace images, 600 ECG trace images of abnormal heartbeats patients and 300 ECG trace images of myocardial infarction patients are used for training and test of the CNN model. For this task, there are 2170 ECG trace images in total and these images are randomly separated as training, validation and test sets having the ratio of 60:20:20.

Because the CNN models are like black boxes, it is quite unclear how and what features are learned by $\mathrm{CNN}$ without displaying the activations of convolutional layers. Figure 3 demonstrates the activations of first convolutional layer. Simple features such as edges and colors are learned and

Table 3 The structure of the proposed network

\begin{tabular}{llll}
\hline & CNN layer & Output shape & Parameter \# \\
\hline 1 & Input & $224 \times 224 \times 3$ & 0 \\
2 & Convolutional & $222 \times 222 \times 96$ & 2688 \\
3 & ReLU & $222 \times 222 \times 96$ & 0 \\
4 & Normalization & $222 \times 222 \times 96$ & 0 \\
5 & Max pooling & $109 \times 109 \times 96$ & 0 \\
6 & Convolutional & $111 \times 111 \times 192$ & 83,136 \\
7 & ReLU & $111 \times 111 \times 192$ & 0 \\
8 & Max pooling & $54 \times 54 \times 192$ & 0 \\
9 & Convolutional & $56 \times 56 \times 256$ & 221,440 \\
10 & ReLU & $56 \times 56 \times 256$ & 0 \\
11 & Max pooling & $25 \times 25 \times 96$ & 0 \\
12 & Convolutional & $27 \times 27 \times 256$ & 295,168 \\
13 & ReLU & $27 \times 27 \times 256$ & 0 \\
14 & Max pooling & $11 \times 11 \times 256$ & 0 \\
15 & Flatten & $1 \times 1 \times 30976$ & 0 \\
16 & FC & $1 \times 1 \times 4096$ & $126,881,792$ \\
17 & ReLU & $1 \times 1 \times 4096$ & 0 \\
18 & Dropout & $1 \times 1 \times 4096$ & 0 \\
19 & FC & $1 \times 1 \times 2$ & 8194 \\
20 & Softmax & $1 \times 1 \times 2$ & 0 \\
21 & Classification & - & 0 \\
\hline & & &
\end{tabular}

Fig. 3 Activations of convolutional layer

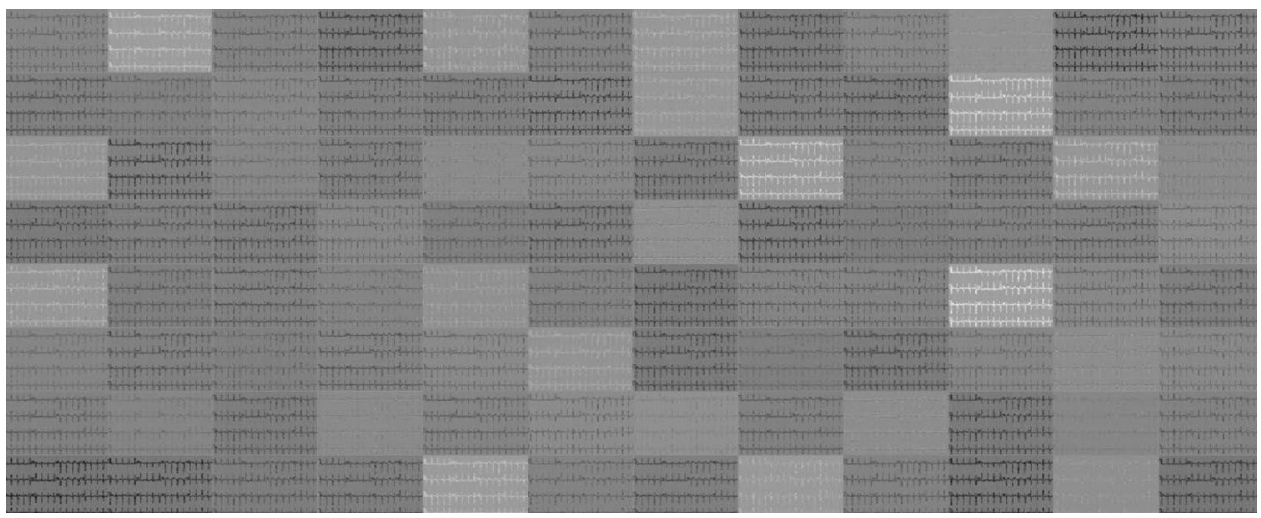


activated in the first convolutional layers whereas more complicated features, which are characteristic key features for the last classification decision, are learned and activated in the deeper convolutional layers. The structure of the proposed CNN architecture is shown in Table 3.

Accuracy and Loss plot results for each classification task are shown in Fig. 4. The proposed method achieves an overall accuracy of $98.57 \%, 93.20 \%, 96.74 \%, 86.55 \%$ and $83.05 \%$ for COVID-19 vs. Normal, COVID-19 vs. Abnormal Heartbeats, COVID-19 vs. Myocardial Infarction binary classification tasks and COVID-19 vs. Abnormal Heartbeats vs. Myocardial Infarction and Normal vs. COVID-19 vs. Abnormal Heartbeats vs. Myocardial Infarction tasks as shown in Fig. 4a, b, c, d and e, respectively. Figure 5 shows Confusion Matrix results for each classification task whereas Fig. 6 shows ROC curves results for each classification task. AUC values of $0.9966,0.9921$ and 0.9771 are obtained for COVID-19 vs. Normal, COVID-19 vs. Abnormal Heartbeats and COVID-19 vs. Myocardial Infarction binary classification tasks, respectively.

All the experimental results for each classification task are collected in Table 4. Classification performance for each task
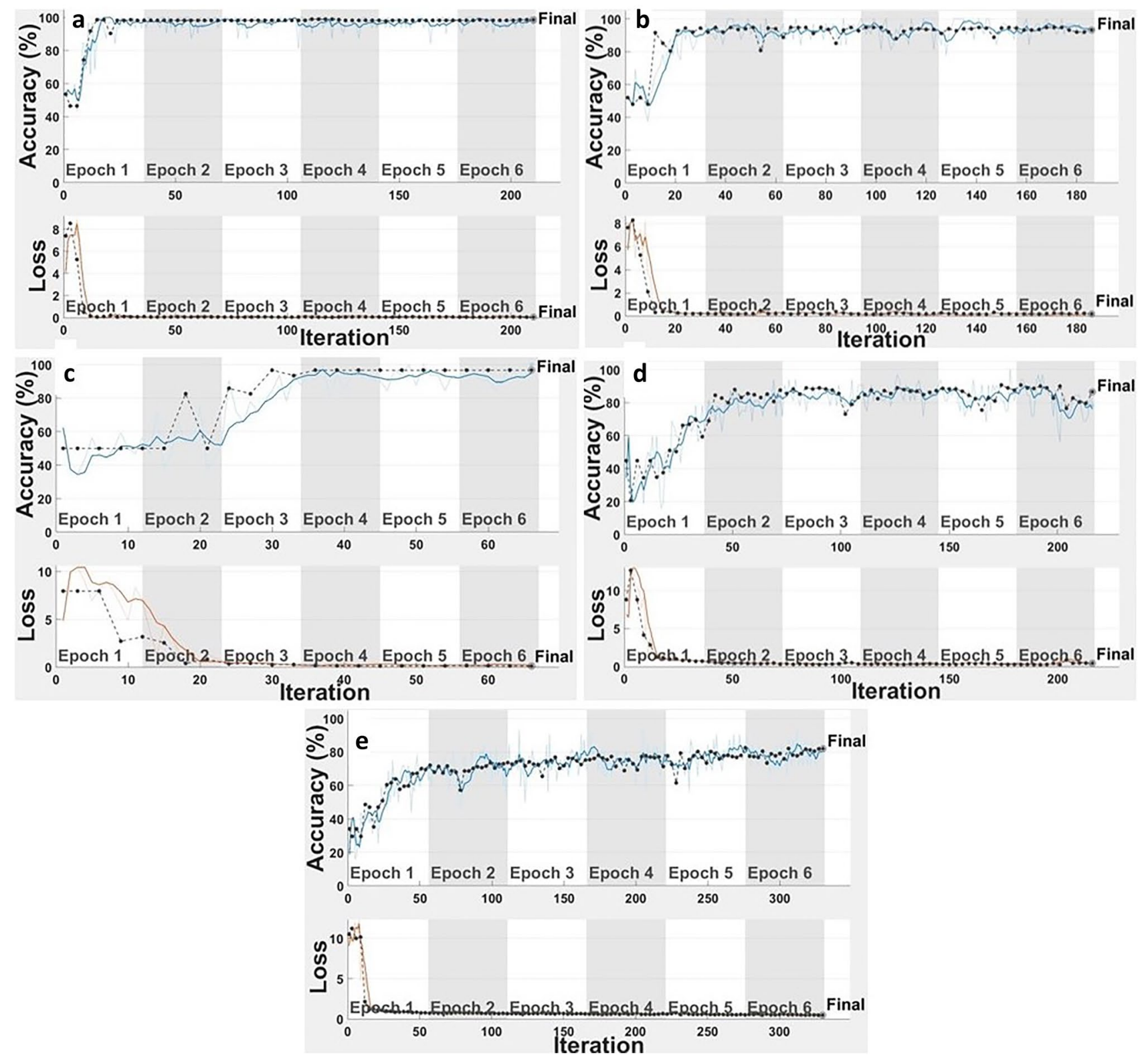

Fig. 4 Accuracy and Loss plot for classification of a COVID-19 vs. Normal, b COVID-19 vs. Abnormal Heartbeats, c COVID-19 vs. Myocardial Infarction, d COVID-19 vs. Abnormal Heartbeats vs.
Myocardial Infarction and e Normal vs. COVID-19 vs. Abnormal Heartbeats vs. Myocardial Infarction 


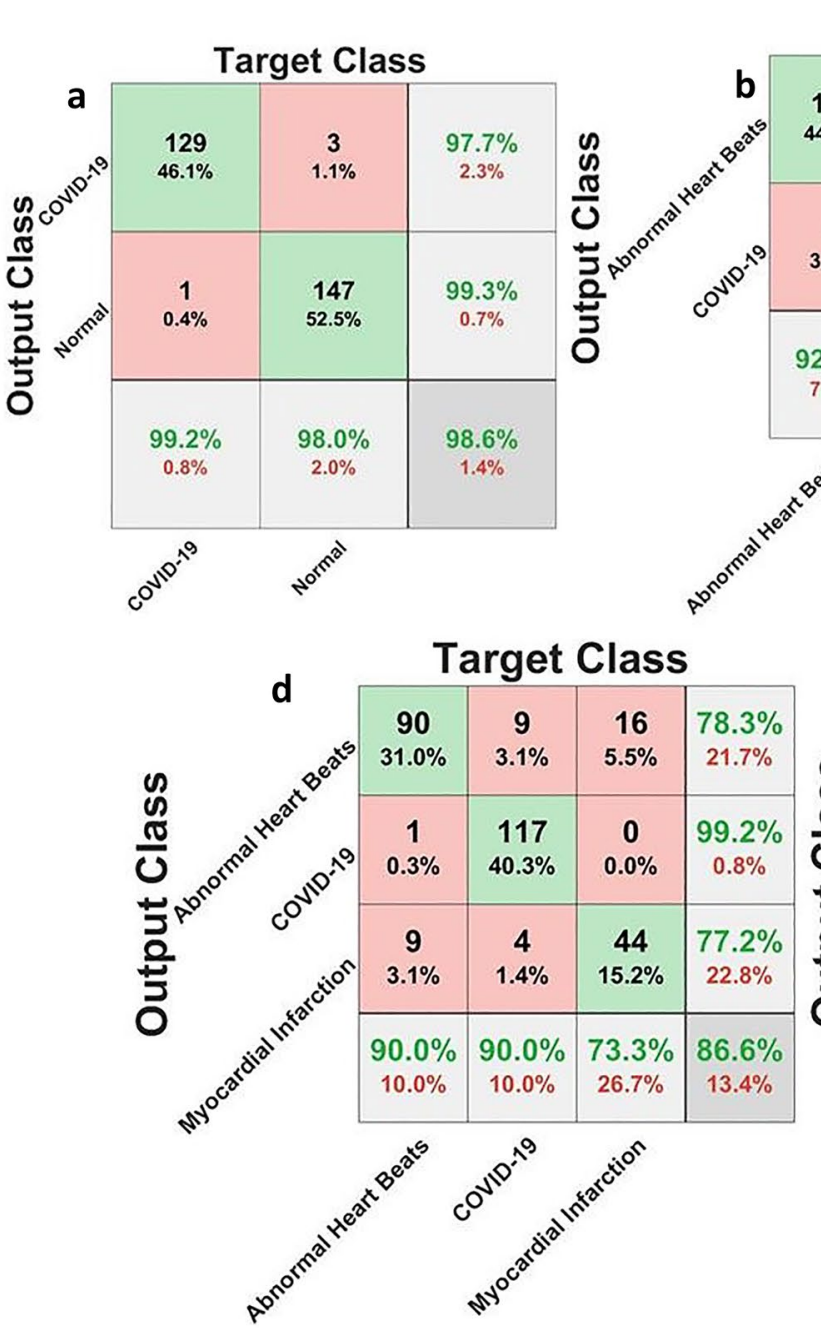

Fig. 5 Confusion Matrix for classification of a COVID-19 vs. Normal, b COVID-19 vs. Abnormal Heartbeats, c COVID-19 vs. Myocardial Infarction, d COVID-19 vs. Abnormal Heartbeats vs. Myocar-

is evaluated for each fold, and the average classification performance of the model is calculated. The proposed CNN model can distinguish COVID-19 disease from Normal, Abnormal Heartbeats and Myocardial Infarction cases with high accuracies of $98.57 \%, 93.20 \%, 96.74 \%$ and AUC value of 0.9966 , $0.9771,0.9905$, respectively. The proposed model is also capable of differentiate between COVID-19, Abnormal Heartbeats and Myocardial Infarction with a satisfactory classification rate of $86.55 \%$ and between Normal vs. COVID-19, Abnormal Heartbeats and Myocardial Infarction with a satisfactory classification rate of $83.05 \%$.

\section{Target Class}
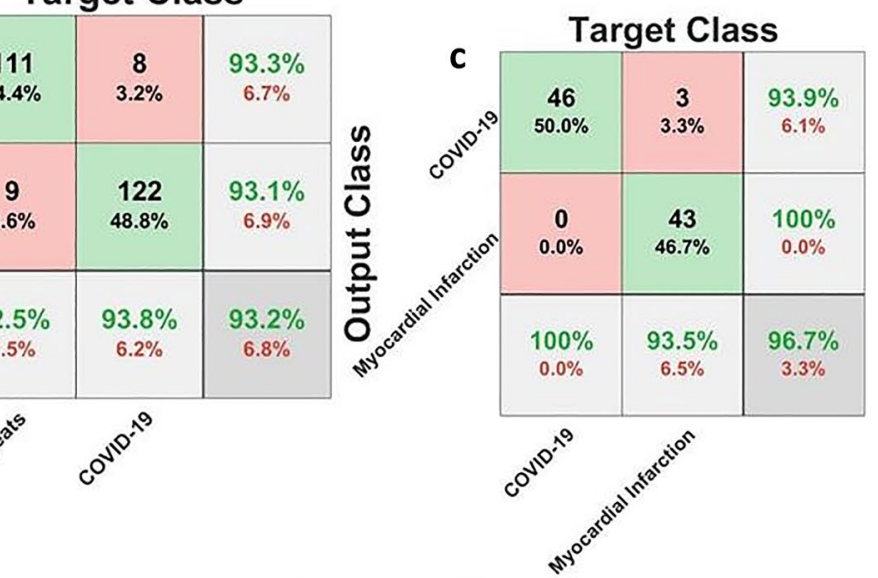

Target Class

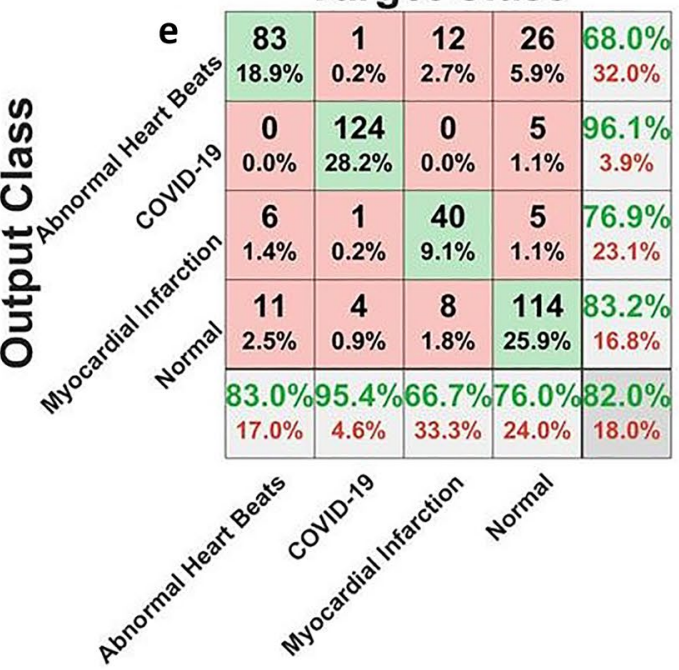

dial Infarction and e Normal vs. COVID-19 vs. Abnormal Heartbeats vs. Myocardial Infarction

\section{Discussion}

Although more than four million people worldwide have already died from COVID-19 disease, the virus still continues to spread very rapidly especially due to the new variants. RT-PCR tests, which have been used for the diagnosis of the disease from the first moment the virus emerged, are still the most widely used diagnostic method in practice today. It is known that the success rate of these tests is between 60 and $70 \%$ which is pretty low to fight against 

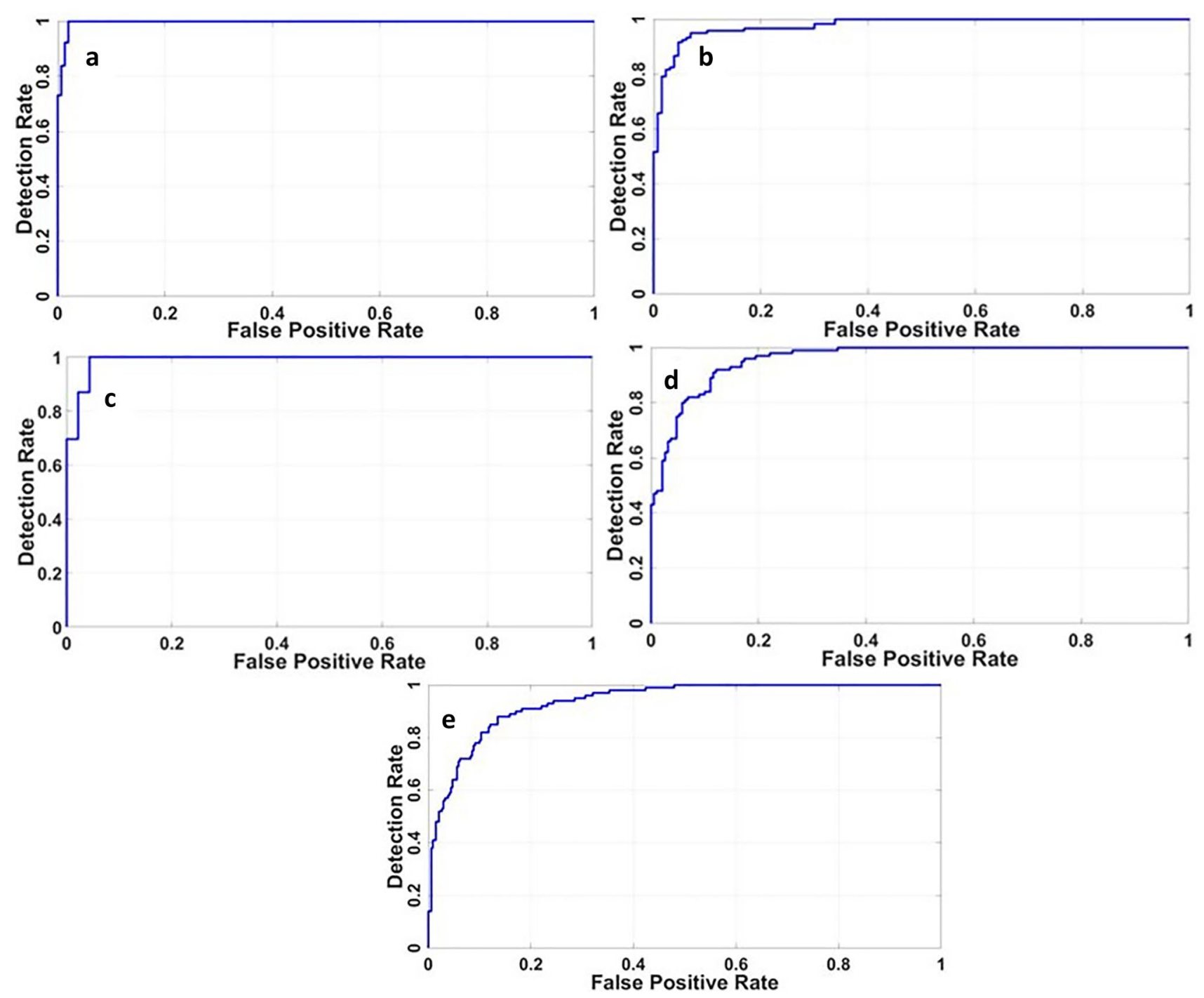

Fig. 6 ROC Curve for classification of a COVID-19 vs. Normal, b COVID-19 vs. Abnormal Heartbeats, c COVID-19 vs. Myocardial Infarction, d COVID-19 vs. Abnormal Heartbeats vs. Myocardial

the highly contagious disease. Intensive scientific studies conducted to diagnose COVID-19 disease with a higher success rate have given birth to radiological imaging based diagnostic methods. It was found that the disease could be diagnosed with higher accuracy with radiological imaging methods. As a result, especially DL-based image processing methods have been developed for COVID-19 disease diagnosis. Despite the high success of radiological imaging methods, these methods have not replaced standard RT-PCR tests for COVID-19 disease diagnosis. The lack of sufficient amount of radiological imaging devices, their expensiveness, and slow results compared to the spread of the pandemic are among the main reasons for this consequence. The current situation clearly shows the necessity of a fast, reliable, inexpensive, harmless and easily
Infarction and e Normal vs. COVID-19 vs. Abnormal Heartbeats vs. Myocardial Infarction

accessible method for the diagnosis of COVID-19 disease. Despite the excellent advances of artificial intelligence, machine learning, deep learning and ECG tools in medicine in recent years, especially regarding diagnosis and treatment, the diagnosis of COVID-19 disease from ECG data using deep learning methods is still far behind. In this study, the diagnosis of COVID-19 disease is successfully performed using a novel deep CNN model by using only paper-based ECG trace images created from ECG signals of COVID-19 infected patients based on the abnormalities caused by the COVID-19 virus on the cardiovascular system. An overall classification accuracy of $98.57 \%$, 93.20\%, 96.74\% and AUC value of 0.9966, 0.9771, 0.9905 is achieved for COVID-19 vs. Normal, COVID-19 vs. Abnormal Heartbeats and COVID-19 vs. Myocardial 
Table 4 Performance evaluation metrics in terms of TP, TN, FP, FN, accuracy, AUC, specificity, sensitivity and precision

$\begin{array}{llll}\begin{array}{l}\text { COVID-19 vs } \\ \text { normal }\end{array} & \begin{array}{l}\text { COVID-19 vs. } \\ \text { abnormal heartbeats }\end{array} & \begin{array}{l}\text { COVID-19 vs. } \\ \text { myocardial infarc- } \\ \text { tion }\end{array} & \begin{array}{l}\text { COVID-19 vs. abnormal } \\ \text { heartbeats vs. myocardial } \\ \text { infarction }\end{array}\end{array}$

\begin{tabular}{|c|c|c|c|c|c|c|c|c|c|c|c|c|c|}
\hline \multirow[b]{2}{*}{$\mathrm{TP}$} & \multicolumn{2}{|l|}{$\begin{array}{l}\text { COVID-19 vs } \\
\text { normal }\end{array}$} & \multicolumn{2}{|c|}{$\begin{array}{l}\text { COVID-19 vs. } \\
\text { abnormal heartbeats }\end{array}$} & \multicolumn{2}{|c|}{$\begin{array}{l}\text { COVID-19 vs. } \\
\text { myocardial infarc- } \\
\text { tion }\end{array}$} & \multicolumn{3}{|c|}{$\begin{array}{l}\text { COVID-19 vs. abnormal } \\
\text { heartbeats vs. myocardial } \\
\text { infarction }\end{array}$} & \multicolumn{4}{|c|}{$\begin{array}{l}\text { Normal vs. COVID-19 } \\
\text { vs. abnormal heartbeats } \\
\text { vs. myocardial infarc- } \\
\text { tion }\end{array}$} \\
\hline & 129 & 147 & 122 & 111 & 46 & 43 & 117 & 90 & 44 & 114 & 124 & 83 & 40 \\
\hline $\mathrm{TN}$ & 147 & 129 & 111 & 122 & 43 & 46 & 159 & 165 & 217 & 267 & 305 & 301 & 368 \\
\hline FP & 3 & 1 & 9 & 8 & 3 & 0 & 1 & 25 & 13 & 23 & 5 & 39 & 12 \\
\hline $\mathrm{FN}$ & 1 & 3 & 8 & 9 & 0 & 3 & 13 & 10 & 16 & 36 & 6 & 17 & 20 \\
\hline Total & 130 & 150 & 130 & 120 & 46 & 46 & 130 & 100 & 60 & 150 & 130 & 100 & 60 \\
\hline Accuracy $\% \pm \mathrm{CI} \%$ & $98.57 \pm 1.14$ & & $93.20 \pm 2.73$ & & $96.74 \pm 3.54$ & & $86.55 \pm 3.47$ & & & 83.0 & \pm 3.96 & & \\
\hline $\operatorname{AUC}(\%)$ & $99.66 \pm 0.26$ & & $97.71 \pm 1.82$ & & $99.05 \pm 0.68$ & & NA & & & NA & & & \\
\hline Specificity (\%) & $98.00 \pm 1.29$ & & $92.50 \pm 3.39$ & & $93.48 \pm 5.10$ & & $99.38 \pm 0.76$ & & & 98.3 & \pm 1.64 & & \\
\hline Sensitivity (\%) & $99.23 \pm 0.59$ & & $93.85 \pm 3.99$ & & $99.89 \pm 0.60$ & & $90.00 \pm 6.27$ & & & 95.3 & \pm 3.59 & & \\
\hline Precision (\%) & $97.73 \pm 1.91$ & & $93.13 \pm 4.24$ & & $93.88 \pm 4.20$ & & $99.15 \pm 0.87$ & & & 96.1 & \pm 3.59 & & \\
\hline
\end{tabular}

CI $95 \%$ confidence interval, $A U C$ area under the curve, $T P$ true positive, $T N$ true negative, $F P$ false positive, $F N$ false negative

Infarction binary classification tasks, respectively. In addition, an overall classification accuracy $86.55 \%$ and $83.05 \%$ is achieved for COVID-19 vs. Abnormal Heartbeats vs. Myocardial Infarction and Normal vs. COVID-19 vs. Abnormal Heartbeats vs. Myocardial Infarction multiclassification tasks, respectively. There are only two quite similar previous studies considering the latest literature. In the first study, COVID-19 disease has been performed from ECG data using well-known pre-trained DL models such as InceptionV3, MobileNetv2, DenseNet201, ResNet18, ResNet50 and ResNet101 [26]. The experimental results show that the pre-trained DenseNet201 outperforms other CNN models for COVID-19 vs. Normal binary classification with an accuracy of $99.1 \%$. In addition to this, DenseNet201 outperforms other CNN models for COVID-19 vs. Normal vs. Other Cardiovascular diseases three-classification task with an accuracy of $97.36 \%$. On the other hand, InceptionV3 outperforms other CNN models for COVID-19 vs. Normal vs. Myocardial Infarction vs Abnormal Heartbeats vs. Recovered Myocardial Infarction multi-classification task with an accuracy of $97.83 \%$. In the second study, the possibility of diagnosing COVID19 disease from ECG data has been investigated using a novel CNN model [27]. In the aforementioned study, the proposed method has achieved an accuracy rate of $81.08 \%$ for COVID-19 vs. Normal binary classification using ECG trace images. Only 250 ECG trace images of COVID-19 patients and 83 ECG trace images of normal people have been used for training and test of CNN model.

There are successful studies about COVID-19 disease diagnosis from radiological imaging using DL based image processing methods. For example, Ozturk et al. have used the DarkNet model as a classifier for the you only look once (YOLO) real time object detection system to predict COVID-19 disease from chest X-ray images with an accuracy of $98.08 \%$ [40]. In another study Apostolopoulos et al. have achieved $96.78 \%$ accuracy for COVID19 disease diagnosis from chest X-ray images using transfer learning technique of DL phenomenon [17]. Irmak E. has also used chest X-ray images and a novel CNN model for the diagnosis of COVID-19 disease with an satisfactory accuracy rate of $98.92 \%$ [7]. Togacar et al. have achieved 99.27\% diagnosis rate for COVID-19 disease using DL models. Ismael et al. have introduced a DL based approach for the COVID-19 diagnosis using chest X-ray images obtaining an accuracy of $94.7 \%$ [41]. Pathak et al. have obtained accuracy of $93.02 \%$ in COVID-19 disease diagnosis using Deep Transfer Learning approach [42]. There are also studies that used chest CT images for COVID-19 disease diagnosis. For instance, $\mathrm{Li}$ et al. have proposed a DL based method for quantitative analysis of COVID-19 disease diagnosis [23]. Chen et al. have studied COVID-19 disease diagnosis from chest CT images using DL based model and obtained $96 \%$ accuracy rate. Song et al. have developed a DL based CT diagnosis system for COVID-19 disease diagnosis and achieved an accuracy value of $86 \%$. Amyar et al. were another researcher group who accurately diagnosed COVID-19 disease from CT image using DL based image processing model with an accuracy rate of $94.67 \%$ [43].

There are well-known existing popular pre-trained CNN models which have been trained on different classification purposes in the literature. It is worth comparing the results obtained by the proposed $\mathrm{CNN}$ model in this paper with the results of those existing popular state-of-the-art CNN models. Therefore, the same experiments with the same dataset are conducted using the popular well-known pre-trained CNN models such as ResNet-50, ResNet-101, DenseNet, InceptionV3, VGG-16 and VGG-19. The results are demonstrated in Table 5. 
Table 5 Overall accuracy (\%) results of different classification tasks for different well-known CNN models

\begin{tabular}{llllll}
\hline & COVID-19 vs normal & $\begin{array}{l}\text { COVID-19 vs } \\
\text { abnormal heart- } \\
\text { beats }\end{array}$ & $\begin{array}{l}\text { COVID-19 } \\
\text { vs myocardial } \\
\text { infarction }\end{array}$ & $\begin{array}{l}\text { COVID-19 vs abnormal } \\
\text { heartbeats vs. myocardial } \\
\text { infarction }\end{array}$ & $\begin{array}{l}\text { Normal vs COVID-19 vs } \\
\text { abnormal heartbeats vs myo- } \\
\text { cardial infarction }\end{array}$ \\
\hline ResNet-50 & $96.22 \pm 1.41$ & $91.52 \pm 2.72$ & $95.91 \pm 3.50$ & $83.45 \pm 5.04$ & $78.08 \pm 3.21$ \\
ResNet-101 & $97.43 \pm 2.10$ & $92.60 \pm 4.51$ & $94.43 \pm 4.21$ & $82.51 \pm 4.66$ & $80.76 \pm 9.53$ \\
DenseNet & $96.27 \pm 2.48$ & $89.72 \pm 6.05$ & $93.92 \pm 5.85$ & $86.38 \pm 4.35$ & $76.83 \pm 4.87$ \\
InceptionV3 & $95.90 \pm 3.51$ & $91.67 \pm 5.67$ & $92.86 \pm 8.93$ & $87.61 \pm 4.12$ & $79.35 \pm 4.23$ \\
VGG-16 & $97.79 \pm 2.00$ & $92.07 \pm 3.97$ & $95.29 \pm 4.75$ & $88.75 \pm 3.45$ & $83.74 \pm 3.38$ \\
VGG-19 & $96.71 \pm 4.01$ & $91.80 \pm 4.86$ & $94.14 \pm 4.21$ & $86.95 \pm 11.17$ & $83.32 \pm 11.15$ \\
Proposed model & $98.57 \pm 1.41$ & $93.20 \pm 2.73$ & $96.74 \pm 2.54$ & $86.55 \pm 2.47$ & $83.05 \pm 2.96$ \\
\hline
\end{tabular}

Considering the experimental results, discussions, literature survey etc. the main contribution of the proposed study in this paper can be summarized as follows:

- The diagnosis of COVID-19 disease is successfully performed using a novel deep CNN model by using only ECG trace images created from ECG signals of COVID19 infected patients based on the abnormalities caused by the COVID-19 virus on the cardiovascular system.

- The proposed method achieves an overall accuracy of $98.57 \%, 93.20 \%, 96.74 \%, 86.55 \%$ and $83.05 \%$ for COVID-19 vs. Normal, COVID-19 vs. Abnormal Heartbeats, COVID-19 vs. Myocardial Infarction binary classification tasks and COVID-19 vs. Abnormal Heartbeats vs. Myocardial Infarction and Normal vs. COVID-19 vs. Abnormal Heartbeats vs. Myocardial Infarction multiclassification tasks, respectively.

- Although the main focus of this study is the diagnosis of COVID-19 disease, other cardiovascular diseases such as, Abnormal Heartbeats, Myocardial Infarction are also successfully diagnosed using CNN method.

- The proposed method in this paper is believed to be an alternative high-accurate and effective method to fill the gap for COVID-19 disease diagnosis taking into account the promising ECG features such as accessibility, reliability, low cost, real-time monitoring, harmlessness etc.

There are of course some limitations of this study. Despite the fact that the publicly available dataset used in this study includes ECG data of different patients, the proposed study needs to be assessed with different datasets. The fact that the dataset used in this study is the only publicly available paper-based ECG trace images data source shows the importance of sharing more ECG data to further develop diagnostic and treatment methods. Another concern is the ambiguity which has been emphasized in some COVID-19 reports that can be briefly stated that the changes in the cardiovascular system may not be caused by the COVID-19 disease alone. Other upcoming medical studies on COVID-19 disease and sharing more datasets will eliminate these concerns and limitations.

\section{Conclusion}

In this study, it is aimed to make a fast and accurate diagnosis of COVID-19 disease from the ECG trace images of patients with the DL based CNN method, based on the scientific reports revealing that the COVID-19 disease causes changes in the cardiovascular system. A novel CNN model successfully differentiates COVID-19 cases from Normal, Abnormal Heartbeats and Myocardial cases with overall accuracy of $98.57 \%, 93.20 \%$ and $96.74 \%$, respectively. In addition, the proposed model can differentiate between COVID-19, Abnormal Heartbeats and Myocardial Infarction with an overall accuracy of $86.55 \%$. Moreover, it can differentiate between Normal, COVID-19, Abnormal Heartbeats and Myocardial Infarction with an overall accuracy of $83.05 \%$. The proposed method outperforms the wellknown CNN models and achieves promising performance. Although there are many successful studies for the diagnosis of COVID-19 disease from radiological images, this fully automatic proposed study is much more advantageous in the setting of the pandemic as it uses ECG data, which is faster, easier and cheaper to access the same diagnosis compared to radiological images. In future work, the diagnostic method proposed in this study has the potential to be actively used during pandemic periods by being installed on mobile phones together with real-time cloud-based systems.

Author contributions EI conceived and designed research; performed experiments; analyzed data; interpreted results of experiments; prepared figures.

Funding The author received no financial support for the research, authorship, and/or publication of this article.

Availability of data and material Not applicable. 
Code availability Not applicable.

\section{Declarations}

Conflict of interest The author declares that there is no conflict of interest regarding the publication of this article.

Ethical statement This study was performed in line with the principles of the Declaration of Helsinki.

\section{References}

1. Di Gennaro F et al (2020) Coronavirus diseases (COVID-19) current status and future perspectives: a narrative review. Int J Environ Res Public Health. https://doi.org/10.3390/ijerph17082690

2. John Hopkins University Coronavirus Resource Center. https:// coronavirus.jhu.edu/map.html. Accessed 16 June 2021

3. World Health Organization. https://www.who.int/. Accessed 16 June 2021

4. Our world in data. https://ourworldindata.org. Accessed 3 June 2021

5. Li L et al (2020) Using artificial intelligence to detect COVID19 and community-acquired pneumonia based on pulmonary CT: Evaluation of the diagnostic accuracy. Radiology 296(2):E65E71. https://doi.org/10.1148/radiol.2020200905

6. Wong HYF et al (2020) Frequency and distribution of chest radiographic findings in patients positive for COVID-19. Radiology 296(2):E72-E78. https://doi.org/10.1148/radiol.2020201160

7. Irmak E (2020) Implementation of convolutional neural network approach for COVID-19 disease detection. Physiol Genom 52(12):590-601. https://doi.org/10.1152/physiolgenomics.00084. 2020

8. Irmak E (2020) A novel deep convolutional neural network model for COVID-19 disease detection. In: Medical Technologies National Conference (TIPTEKNO), no 11, pp 39-42

9. Irmak E (2021) COVID-19 disease severity assessment using CNN model. IET Image Process 15(8):1814-1824. https://doi. org/10.1049/ipr2.12153

10. Jain U (2020) Effect of COVID-19 on the organs. Cureus 12(8):18. https://doi.org/10.7759/cureus. 9540

11. Capaccione KM et al (2021) Pathophysiology and imaging findings of COVID-19 infection: an organ-system based review. Acad Radiol 28(5):595-607. https://doi.org/10.1016/j.acra.2021.01.022

12. Bergamaschi $L$ et al (2021) The value of ECG changes in risk stratification of COVID-19 patients. Ann Noninvasive Electrocardiol 26(3):1-10. https://doi.org/10.1111/anec.12815

13. Nemati R, Ganjoo M, Jadidi F, Tanha A, Baghbani R (2020) Electrocardiography in early diagnosis of cardiovascular complications of COVID-19; a systematic literature review. Arch Acad Emerg Med 9(1):1-10. https://doi.org/10.22037/aaem.v9i1.957

14. Mehraeen E et al (2020) A systematic review of ECG findings in patients with COVID-19. Indian Heart J 72(6):500-507. https:// doi.org/10.1016/j.ihj.2020.11.007

15. Angeli F et al (2020) Electrocardiographic features of patients with COVID-19 pneumonia. Eur J Intern Med. https://doi.org/10. 1016/j.ejim.2020.06.015

16. Toğaçar M, Ergen B, Cömert Z (2020) COVID-19 detection using deep learning models to exploit Social Mimic Optimization and structured chest X-ray images using fuzzy color and stacking approaches. Comput Biol Med 121(6):1-12. https://doi.org/10. 1016/j.compbiomed.2020.103805
17. Ioannis A, Mpesiana T (2020) Covid-19: automatic detection from X-ray images utilizing transfer learning with convolutional neural networks. Phys Eng Sci Med 43(2):635-640. https://doi. org/10.1007/s13246-020-00865-4

18. Loey M, Smarandache F, Khalifa NE (2020) Within the lack of chest COVID-19 X-ray dataset: a novel detection model based on GAN and deep transfer learning. Symmetry 12(4):1-19. https://doi.org/10.3390/SYM12040651

19. Singh D, Kumar V, Vaishali, Kaur M (2020) Classification of COVID-19 patients from chest CT images using multiobjective differential evolution-based convolutional neural networks. Eur J Clin Microbiol Infect Dis. https://doi.org/10. 1007/s10096-020-03901-z

20. Chen J et al (2020) Deep learning-based model for detecting 2019 novel coronavirus pneumonia on high-resolution computed tomography: a prospective study. medRxiv. https://doi. org/10.1101/2020.02.25.20021568

21. Chaganti S et al (2020) Automated quantification of CT patterns associated with COVID-19 from chest CT. Radiol Artif Intell 2(4):e200048. https://doi.org/10.1148/ryai.2020200048

22. Chassagnon $\mathrm{G}$ et al (2021) AI-driven quantification, staging and outcome prediction of COVID-19 pneumonia. Med Image Anal 67:101860. https://doi.org/10.1016/j.media.2020.101860

23. Li $\mathrm{Z}$ et al (2020) From community-acquired pneumonia to COVID-19: a deep learning-based method for quantitative analysis of COVID-19 on thick-section CT scans. Eur Radiol 30(12):6828-6837. https://doi.org/10.1007/ s00330-020-07042-x

24. Huang L et al (2020) Serial quantitative chest CT assessment of COVID-19: a deep learning approach. Radiol Cardiothorac Imaging 2(2):1-8. https://doi.org/10.1148/ryct.2020200075

25. Zhu J, Shen B, Abbasi A, Hoshmand M, Li H, Duong T (2020) Deep transfer learning artificial intelligence accurately stages COVID-19 lung disease severity on portable chest radiographs. PLoS ONE 15(7):1-11. https://doi.org/10.1371/journal.pone. 0236621

26. Ozdemir MA, Ozdemir GD, Guren O (2021) Classification of COVID-19 electrocardiograms by using hexaxial feature mapping and deep learning. BMC Med Inform Decis Mak 21(1):1-20. https://doi.org/10.1186/s12911-021-01521-x

27. Rahman $\mathrm{T}$ et al (2021) COV-ECGNET: COVID-19 detection using ECG trace images with deep convolutional neural network. arXiv (Preprint)

28. Shi S et al (2020) Characteristics and clinical significance of myocardial injury in patients with severe coronavirus disease 2019. Eur Heart J 41(22):2070-2079. https://doi.org/10.1093/eurheartj/ ehaa408

29. He J et al (2020) Characteristic electrocardiographic manifestations in patients with COVID-19. Can J Cardiol 36(6):996.e1-996. e4. https://doi.org/10.1016/j.cjca.2020.03.028

30. Churchill TW et al (2020) Echocardiographic features of COVID19 illness and association with cardiac biomarkers. J Am Soc Echocardiogr 33(8):1053-1054. https://doi.org/10.1016/j.echo. 2020.05.028

31. Khan AH, Hussain M, Malik MK (2021) ECG images dataset of cardiac and COVID-19 patients. Data Br 34:106762. https://doi. org/10.1016/j.dib.2021.106762

32. Alom MZ et al (2019) A state-of-the-art survey on deep learning theory and architectures. Electronics 8(3):1-67. https://doi.org/ 10.3390/electronics 8030292

33. Irmak E (2021) A novel implementation of deep-learning approach on malaria parasite detection from thin blood cell images. Electrica 21(2):216-224. https://doi.org/10.5152/elect rica.2020.21004

34. Irmak E (2021) Multi-classification of brain tumor MRI images using deep convolutional neural network with fully optimized 
framework. Iran J Sci Technol Trans Electr Eng 45(3):1015-1036. https://doi.org/10.1007/s40998-021-00426-9

35. Simonyan K, Zisserman A (2015) Very deep convolutional networks for large-scale image recognition. The 3rd International Conference on Learning Representations (ICLR2015), San Diego. https://arxiv.org/abs/1409.1556

36. Kingma DP, Ba LJ (2015) Adam: a method for stochastic optimization. The 3rd International Conference on Learning Representations (ICLR2015), San Diego. http://arxiv.org/abs/1412.6980

37. Radiuk P (2017) Impact of training set batch size on the performance of convolutional neural networks for diverse datasets. Inf Technol Manag Sci 20:20-24. https://doi.org/10.1515/ itms-2017-0003

38. Kandel I, Castelli M (2020) The effect of batch size on the generalizability of the convolutional neural networks on a histopathology dataset. ICT Express 6(4):312-315. https://doi.org/10.1016/j. icte.2020.04.010

39. Tilborghs S et al (2020) Comparative study of deep learning methods for the automatic segmentation of lung, lesion and lesion type in CT scans of COVID-19 patients. arXiv (Preprint)

40. Ozturk T, Talo M, Yildirim EA, Baloglu UB, Yildirim O, RajendraAcharya U (2020) Automated detection of COVID-19 cases using deep neural networks with X-ray images. Comput Biol Med 121(April):103792. https://doi.org/10.1016/j.compbiomed.2020. 103792

41. Ismael AM, Şengür A (2021) Deep learning approaches for COVID-19 detection based on chest X-ray images. Expert Syst Appl 164:114054. https://doi.org/10.1016/j.eswa.2020.114054

42. Pathak Y, Shukla PK, Tiwari A, Stalin S, Singh S, Shukla PK (2020) Deep transfer learning based classification model for COVID-19 disease. IRBM. https://doi.org/10.1016/j.irbm.2020. 05.003 (in press)

43. Amyar A, Modzelewski R, Li H, Ruan S (2020) Multi-task deep learning based CT imaging analysis for COVID-19 pneumonia: classification and segmentation. Comput Biol Med 126:104037. https://doi.org/10.1016/j.compbiomed.2020.104037

Publisher's Note Springer Nature remains neutral with regard to jurisdictional claims in published maps and institutional affiliations. 